\title{
Respuesta educativa en la atención a la diversidad desde la perspectiva de profesionales de apoyo
}

Educational Response in the Attention to Diversity from the Perspective of Support Professionals

Resposta da educação na atenção à diversidade na perspectiva de profissionais de suporte

\section{Carolina Cornejo-Valderrama* (iD) o000-0003-3504-4540}

Artículo de investigación

Revista Colombiana de Educación, N.7 73. Segundo semestre de 2017, Bogotá, Colombia.

doi: 10.17227/01203916.73rce75.94

Para citar este artículo: Cornejo, C. (2Ol7). Respuesta educativa en la atención a la diversidad desde la perspectiva de profesionales de apoyo. Revista Colombiana de Educación,(73), 77-96.

\section{(C) $(1) \Theta$}




\section{Resumen}

El propósito de este artículo es analizar las respuestas educativas en la atención a la diversidad que plantean 92 profesionales especialistas de apoyo que se desempeñan en 30 establecimientos educativos públicos de Chile que forman parte del Programa de Integración Escolar. Los participantes son profesores de educación especial, psicopedagogos, psicólogos, fonoaudiólogos, kinesiólogos, un orientador educacional y un trabajador social que atienden a niños, niñas y jóvenes que presentan necesidades educativas especiales, en la región del Maule. La investigación es cuantitativa de diseño descriptivo, no experimental y transeccional, utilizando la técnica de la encuesta y el análisis de los datos a través de estadígrafos descriptivos y frecuencias. Los resultados muestran que los profesionales de apoyo perciben la variable contexto de manera positiva, y reconocen una desvinculación de las unidades educativas con instituciones externas como centros de apoyo en la atención a la diversidad. Además, los hallazgos revelan la diferencia de paradigmas en la comprensión de los diagnósticos de los educandos, moviéndose entre enfoques clínicos y educativos. Sin embargo, coinciden en señalar la diversidad como algo positivo y enriquecedor, cuando la escuela asume las diferencias individuales como propias, y flexibiliza y adapta el currículo escolar. Es importante destacar que al tensionar los hallazgos encontrados no se relacionan directamente con la mejora de los aprendizajes y la disminución de estudiantes que participan en los programas de integración escolar.

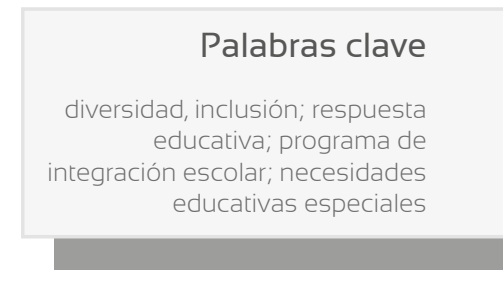

\section{Keywords}

diversity; inclusion; educational response; school integration program; special educational needs

\begin{abstract}
The purpose of this article is to analyze the educational responses in attention to diversity posed by 92 professional support specialists who work in 30 public schools in Chile involved in the School Integration Program. The participants are special education teachers, psychology and pedagogy experts, psychologists, speech therapists, physiotherapists, an educational counselor and a social worker caring for children and young people with special educational needs, in the region of Maule. The research design is quantitative and descriptive, non-experimental and transactional, using the survey technique and data analysis through descriptive statistics and frequencies. The results show that support professionals perceive the context variable positively and recognize a detachment of educational units from external institutions as support centers in the attention to diversity. Furthermore, the findings reveal the difference of paradigms in understanding the diagnoses of students, moving between clinical and educational approaches. However, there is an agreement with the idea that diversity is something positive and enriching, when the school assumes individual differences as its own, and is available to make the curriculum more flexible and to adapt it. It is important to emphasize that when stressing the findings, they do not relate directly to improved learning and decreased students participating in school integration programs.
\end{abstract}

\begin{abstract}
Resumo
O objetivo deste artigo é analisar as respostas educativas em atenção à diversidade propostas por 92 especialistas de suporte profissionais que trabalham em 30 escolas públicas no Chile, as quais formam parte do Programa de Integração Escolar. Os participantes são professores de educação especial, psicólogos, fonoaudiólogos, fisioterapeutas, um conselheiro educacional e um assistente social para crianças e jovens com necessidades educativas especiais na região de Maule. A pesquisa é quantitativa, de caráter descritiva, não experimental e transversal, utilizando a técnica de levantamento e análise de dados através de estatísticas descritivas e frequências. Os resultados mostram que os profissionais de suporte percebem a variável de contexto positivamente e reconhecem a separação de unidades educacionais com instituições externas como centros de apoio na atenção à diversidade. Além disso, os resultados revelam a diferença de paradigmas na compreensão dos diagnósticos de alunos, movendo-se entre as abordagens clínicas e educacionais. No entanto, assinalam a diversidade como algo positivo e enriquecedor se a escola adota as diferenças individuais como próprias, e flexibiliza e adapta o currículo escolar. É importante ressaltar que ao analisar as conclusões, estas não se relacionam diretamente com o melhoramento da aprendizagem e a diminuição dos estudantes que participam nos programas de integração escolar.
\end{abstract}

\section{Palavras chave}

diversidade; inclusão; resposta educativa; programa de integração escolar; necessidades educativas especiais 


\section{Introducción}

En Chile, la educación se enfoca en los principios señalados en la Ley General de Educación n. 20.370 del 2009 del Ministerio de Educación (Mineduc). En ella se señalan, entre otros, los principios de calidad y equidad educativa, integración, interculturalidad y diversidad para todos y todas, incluyendo a aquellos jóvenes que deben enfrentar barreras que obstaculizan su proceso educativo.

Por esta razón, se cuenta con una serie de decretos y reglamentos dictados por el Mineduc, para asegurar el respeto a la diversidad educativa y lograr una formación integral de jóvenes insertos dentro del sistema educativo, que necesitan un apoyo extra para acceder, permanecer y progresar en los procesos de enseñanza y aprendizaje.

Este marco legal regula el sistema escolar, entregando normas claras y específicas que aseguran un servicio educativo de calidad para todos los niños, niñas y jóvenes, independientemente de su condición social, económica, cultural o territorial. Además, establece condiciones y requisitos para la entrega del servicio educativo que realizan las escuelas y liceos del país, con la finalidad de mejorar la calidad de la educación, de manera que se alcancen los objetivos generales y los estándares de aprendizaje que se definan en la forma que establezca la ley, y se flexibilicen ampliamente tanto el currículum como los objetivos para entregar una educación integral a los jóvenes.

Siendo la educación un derecho para todos y todas, las respuestas educativas deben ajustarse a las necesidades de los estudiantes, movilizando significaciones más comprensivas del concepto de inclusión educativa. De este modo, Booth (2002) señala que la inclusión hace referencia al aprendizaje y la participación de todos los educandos, sin distinciones que pudiesen resultar de presiones excluyentes y no exclusivamente para aquellos estudiantes que se encuentran en situación de discapacidad o presentan necesidades educativas especiales.

Siguiendo este planteamiento, toda educación comprensiva e integradora asume la diversidad que presentan los jóvenes, ejerciendo una acción positiva a favor de aquellos que se encuentran en situación de desventaja y requieren de apoyos y ayudas específicas. Además, en este nivel educativo se deben adoptar medidas de flexibilización escolar contextualizadas a las realidades particulares de cada uno de los jóvenes.

De este modo, la atención a la diversidad hace referencia al concepto de inclusión educativa en el sentido más amplio, se la entiende como una reforma que sostiene y acoge la diversidad de todos los educandos (Unesco, 2001). Para ello, se requiere que todos 
... los seres humanos formen sus valores a través de la socialización. Ésta se presenta en dos fases: primaria, en el seno de la familia, y secundaria, en la escuela. Una de las características de la socialización primaria, es la carga afectiva con la que se transmiten los contenidos y la identificación con el mundo tal y como lo presentan los adultos. (Fragoso y Canales, 2009, p. 2).

Cuando niños, niñas y jóvenes se sienten comprometidos con la educación, demuestran interés e inquietud por realizar cambios a nivel social, porque distinguen el efecto sobre su desarrollo y desencadenan estímulos que favorecen el aprendizaje y, por ende, su permanencia y egreso del sistema escolar. Es por esto que los establecimientos educacionales deben ser capaces de adaptarse a la diversidad de estudiantes, entregando herramientas para su desenvolvimiento independiente y autónomo tanto en su entorno inmediato como en la sociedad toda.

La función del Estado es abrir paso a estos principios esenciales, capacitar a docentes y profesionales especialistas que brinden apoyo en el sistema escolar para transmitir a los estudiantes los valores que son base sólida de estos, velando para que en ninguna parte se les ignore, y tengan así el respeto que merecen.

La atención a la diversidad se entiende a nivel general como el conjunto de actuaciones educativas dirigidas a dar respuesta a las diferentes capacidades, ritmos y estilos de aprendizaje, motivaciones e intereses, situaciones sociales, culturales, lingüísticas y de salud del alumnado. Constituye, por tanto, un principio fundamental que debe regir a todos los niveles educativos, asegurando la igualdad de oportunidades de todos los estudiantes ante la educación y evitar, en la medida de lo posible, el fracaso escolar y el consecuente riesgo de abandono del sistema educativo.

La inclusión educativa no se limita a garantizar el acceso de los estudiantes con algún tipo de discapacidad a los establecimientos regulares, sino también a eliminar o minimizar las barreras que limitan el aprendizaje y la participación de toda la población estudiantil. En este mismo sentido, Booth y Ainscow (2000) refuerzan la idea anterior al señalar que

Muchos estudiantes experimentan dificultades porque no se tienen en cuenta sus diferencias en los procesos de enseñanza y aprendizaje. Los diversos grupos sociales, etnias y culturas tienen normas, valores, creencias y comportamientos distintos, que generalmente no forman parte de la cultura escolar, lo que puede limitar sus posibilidades de aprendizaje y de participación, o conducir a la exclusión y discriminación. (p. 7). 


\section{Respuesta educativa en la atención a la diversidad}

En la actualidad, la sociedad insiste en mostrarnos como seres más abiertos a los cambios y en el reconocimiento del pluralismo, de la heterogeneidad y de las diferencias en áreas muy diversas, que van desde la apariencia física hasta los valores. Esta situación influye en muchos aspectos de nuestra vida, especialmente en la educación, que no puede quedar ajena a esos cambios, porque es una parte fundamental de la sociedad, ya que se constituye en un medio y una herramienta para mejorar la calidad de vida de todas las personas.

En este contexto, la escuela como institución educativa es el reflejo de la diversidad, que produce cambios y mejoras de carácter social y cultural; además, replantea la respuesta educativa como una forma de atender las diferencias individuales, que permitan a cada uno de los niños, niñas y jóvenes avanzar y progresar en el sistema escolar, independientemente de sus características y de las necesidades educativas especiales.

La atención a la diversidad surge ante la necesidad de otorgar respuestas educativas adaptadas a las características y necesidades de los educandos, en el afrontamiento a las exigencias escolares. Asimismo, en palabras de Gimeno Sacristán (1999, citado en López Melero, 2004), está en relación con todas las circunstancias o características que hacen que las personas seamos distintas y diferentes. No existe una persona idéntica a otra, aunque dentro de esa diferencia somos a la vez parte de una igualdad que hace que todos y todas formemos parte de la humanidad, a pesar de ser tan distintos unos de otros.

En este mismo sentido, Salinas (2007) lo comprende como "todas aquellas características excepcionales del alumnado provocadas por variados factores que requieren una atención especializada para que todo el alumnado alcance un mismo nivel de aprendizaje" (p. 12). En referencia a la diversidad que se produce en el ámbito educativo, la autora explicita que

Esta tiene su origen en factores diversos como son los factores sociales, económicos, culturales, geográficos y religiosos, así como las diferentes capacidades intelectuales, psíquicas, físicas, sensoriales y motóricas. [Sic] Para atender a tal diversidad, el profesor debe llevar a cabo una serie de proyectos y seguimientos que permitan una mejor convivencia entre la educación orientada a la diversidad y la ordinaria. (p. 12).

En la escuela, la diversidad se percibe como las diferencias personales e individuales que hacen que cada estudiante sea distinto de todos los demás, es decir, sea un ser único e irrepetible. Se destacan las diferencias en ritmos para aprender, los estilos de aprendizajes, los intereses, las creencias, las capacidades e incluso las motivaciones y expectativas que cada niño tiene. 
En Chile, la Ley General de Educación declara la diversidad como uno de los principios de la educación, explicitando que "el sistema debe promover y respetar la diversidad de procesos y proyectos educativos institucionales, así como la diversidad cultural, religiosa y social de las poblaciones que son atendidas por él". (p. 2)

En los establecimientos educacionales este concepto remite al hecho de que todos los estudiantes son diferentes, y presentan características educativas que son compartidas por la mayoría y otras que son propias e individuales. Desde esta perspectiva, la atención educativa a la diversidad comprende todas las acciones que se realizan con el fin de evitar que las diferencias que nos identifican como seres humanos se conviertan en desigualdades. Es adaptar o ajustar la respuesta educativa a las características del que aprende.

En este mismo sentido, Cardona (2006) señala que el término atención a la diversidad es "cualquier decisión que se tome o cualquier actuación que se realice en el ámbito de la educación, al ser la finalidad de dichas actuaciones la atención al alumnado en su unidad y en su diversidad" (p. 77). Al relacionar este planteamiento con lo que ocurre en las escuelas, este tipo de respuesta educativa se asemeja a una enseñanza personalizada que atiende a las necesidades específicas de un colectivo, exigiendo al docente implicado en este proceso conocer y adecuar los contenidos y objetivos de aprendizaje a las características individuales de los estudiantes.

Asimismo, Cabrerizo y Rubio (2007) plantean que

La atención a la diversidad implica, en el plano pedagógico, la necesidad de articular una actuación didáctica que permita atender simultáneamente y con eficacia a unos alumnos que se van diferenciando progresivamente como resultado de la propia escolaridad, de los contextos personales y familiares así como de los intereses que se manifiestan en distintos momentos evolutivos. (p. 49).

Siguiendo el planteamiento anterior, los autores Asencio, Biecinto, García, García, Mafokozi y Ramos (2005/2006) comprenden este concepto como la respuesta educativa que se entrega de manera sistemática a todos los niños y niñas que pueden o no requerir apoyos de carácter transitorio o permanente, favorecen positivamente el desempeño escolar y se asegura el cumplimiento de los principios de equidad y calidad educativa.

Igualmente, Ruiz (2010) explicita que

La atención a la diversidad es un concepto amplio que incluye las dificultades de aprendizaje, discapacidades físicas, psíquicas y sensoriales, los grupos de riesgo, las minorías étnicas, etc. El concepto de diversidad nos plantea que todos los alumnos tienen unas necesidades educativas individuales propias y específicas para poder acceder a las experiencias de aprendizaje necesarias para su socialización, establecidas en el currículo escolar. (p. 2). 
El Mineduc (2008), en el marco de la atención a la diversidad, conduce a los agentes educativos a modificar la dinámica observada en las aulas de clases, a cambiar la perspectiva homogeneizadora que señala que los estudiantes son los que deben adaptarse al currículo. Además, los incentiva a flexibilizar la propuesta curricular para responder a las necesidades educativas de todos los educandos. En este mismo contexto, explicita que

La diversidad de los alumnos y alumnas es consecuencia de su origen social y cultural, y de sus características individuales en cuanto a su historia personal y educativa, capacidades, intereses, motivaciones y estilos de aprendizaje. Esta multiplicidad de factores hace que haya dos alumnos/as idénticos. Sin embargo, a pesar de la constante evidencia de la heterogeneidad de los y las estudiantes, sus familias y los docentes, la educación sigue la tendencia de funcionar con esquemas homogeneizadores, para un "supuesto alumno medio" en cuanto a origen social, cultural y en cuanto a capacidades. (p. 8).

A modo de síntesis de los planteamientos anteriores, se puede explicitar que la atención a la diversidad son las adaptaciones y los apoyos educativos entregados a los estudiantes, de manera temporal o permanente, de acuerdo con las necesidades surgidas desde el contexto en el cual se desenvuelven. Además, es necesario que los profesores tengan siempre presente que el objetivo de una acción educativa diferenciada es adaptar las respuestas a las capacidades y potencialidades de los niños, niñas y jóvenes, para conseguir mayores y mejores aprendizajes de cada uno de ellos.

Los autores Cabrerizo y Rubio (2007) relevan también que el término atención a la diversidad puede ser comprendido como "un principio regulador de la función docente que implica a todas las instancias educativas y que tiene como finalidad aprovechar las peculiaridades de cada alumno para potenciar su desarrollo a través de su aprendizaje" (p.46).

Una de las tareas de los docentes de aula es conocer a cada uno de sus estudiantes, para adaptar las metodologías y tecnologías que van surgiendo en el quehacer profesional, como parte de la entrega de una respuesta educativa adecuada y pertinente. También es importante el apoyo permanente de la familia y del entorno escolar.

Jiménez (2005), en referencia a los beneficios de la adaptación del quehacer educativo de acuerdo con las posibilidades de los estudiantes, plantea:

La experiencia pedagógica señala que cuando los estudiantes de un aula trabajan sobre conceptos y habilidades de una unidad de aprendizaje, sin posibilidad de recibir apoyos o de adaptar algunos elementos de esa unidad, se incrementa el riesgo de que disminuya el rendimiento de alguno de ellos. Por el contrario, en un aula de aprendizaje diferenciado, cuando el profesorado mantiene la referencia de los objetivos 
de curso como medida grupal o interindividual, pero analiza los logros personales en relación con las potencialidades y el punto de partida personal, el rendimiento del alumnado en general, y del que muestra necesidades educativas en particular, se ve incrementado. (p. 34).

La acción educativa que se realiza desde la globalidad y no desde las partes beneficia a todos los estudiantes, en particular a aquellos que presentan necesidades educativas de carácter transitorio o permanente, ampliando las posibilidades de vincularse de mejor manera con los objetivos de aprendizaje a través de metodologías activo-participativas.

Lo anterior permite señalar que

La educación inclusiva no cree en la segregación, ni tampoco considera que haya que hacerle un lugar especial a la niñez con discapacidad. Sino propone que hay un lugar que se llama escuela que es para todos y hay un proceso social Ilamado educación y ese proceso se vive en común. (Ministerio de Educación del Perú, 2006, p. 16).

\section{Programa de Integración Escolar}

La filosofía de la integración escolar se fundamenta en el principio de normalización, de tal manera que lo normal o lo no-normal no se encuentra dentro de la persona, sino fuera de ella, es lo que los demás perciben de ella. De ahí surge como fundamental el cambio de actitud de los individuos y de la sociedad en general frente a una persona más o menos excepcional, y no cambiar a esa persona.

Beeny (1975), respecto de la integración, señala que, como filosofía, significa una valoración de las diferencias humanas. Esto implica aceptarlas como distintos modos de ser dentro de un contexto social y ofrecer, a cada uno de sus miembros, las mejores condiciones para el desarrollo máximo de sus capacidades, colocando a su alcance los mismos beneficios y oportunidades de vida normal.

El Programa de Integración Escolar (PIE), se define como

... una estrategia inclusiva del sistema escolar cuyo propósito es entregar apoyos adicionales (en el contexto del aula común) a los estudiantes que presentan Necesidades Educativas Especiales (NEE), sean éstas de carácter permanente o transitorio, favoreciendo con ello la presencia y participación en la sala de clases, el logro de los objetivos de aprendizaje y la trayectoria educativa de "todos y cada uno de los estudiantes", contribuyendo con ello al mejoramiento continuo de la calidad de la educación en el establecimiento educacional. (Mineduc, 2014, p. 4). 
El proceso de integración instalado en la escuela posibilita al niño desarrollar una vida como ser social, brindándole los medios, recursos y condiciones para participar en el conjunto de actividades escolares. En este contexto, su éxito no depende de la limitación sino de cómo se plantean los factores frente a ella. Por esta razón, la posición integracionista desea provocar un proceso de cambio que no solo se limita al ámbito escolar, sino que se relacionará con cambios fundamentales que involucrarán valores y normas sociales y, por ende, juicios éticos que de ellos se desprenden.

Este programa es un desafío para las escuelas y liceos, ya que se deben mejorar las prácticas pedagógicas por parte de los docentes de aula, para permitir una adecuada y pertinente respuesta en la atención a la diversidad de estudiantes, a través de la utilización de variados recursos, y favorecer el desarrollo de las capacidades y competencias de todos y todas. Además, las unidades educativas deben generar las condiciones y los espacios para la realización del trabajo colaborativo, la aplicación de estrategias de co-enseñanza entre docentes de aula y profesionales de apoyo, asegurando la mejora y el progreso de los aprendizajes.

Por otra parte, brinda una oportunidad de asumir compromisos con los aprendizajes de todos los estudiantes a través de la flexibilización y adecuación del currículo escolar, de procedimientos evaluativos y contextuales, para ofrecer los apoyos específicos que los estudiantes requieren.

Para las familias, asegura apoyo de profesionales y las posibilidades de aprender mejores herramientas que permitan potenciar el aprendizaje escolar de manera armónica y coherente con los planes específicos de apoyo.

El PIE atiende a niños, niñas y jóvenes que presentan algún tipo de necesidad educativa especial, ya sea de carácter transitorio o permanente. A través del Decreto 170 de 2009 se establecen los requisitos que deben cumplir las unidades educativas para implementar este programa y se define al "profesional competente" como aquel profesional autorizado para realizar evaluaciones y diagnósticos, y como un personal de apoyo requerido para la mejora de los aprendizajes. Asimismo, incorpora la entrega de recursos para la atención de estudiantes y contribuye a equiparar las oportunidades de aquellos que presentan NEE, independientemente de sus características individuales y sus condiciones personales.

El Mineduc, a través del Decreto 170 del 2009, define a un alumno o una alumna que presenta NEE, COMO: "Aquel que precisa ayudas y recursos adicionales, ya sean humanos, materiales o pedagógicos, para conducir su proceso de desarrollo y aprendizaje, y contribuir al logro de los fines de la educación" (art.2, literal a).

Asimismo, las clasifica en NEE de carácter permanente y transitorio. Las permanentes son: 
Aquellas barreras para aprender y participar que determinados estudiantes experimentan durante toda su escolaridad como consecuencia de una discapacidad diagnosticada por un profesional competente y que demandan al sistema educacional la provisión de apoyos y recursos extraordinarios para asegurar el aprendizaje escolar. (p. 4).

En el mismo documento se subdividen en discapacidad intelectual, visual, auditiva, multidéficit, disfasia severa y trastorno del espectro autista. Entrega además las conceptualizaciones respectivas para una mejor comprensión e interpretación de cada una de ellas. Del mismo modo, el Mineduc define a un estudiante que presenta NEE de carácter transitorio como:

Aquellas no permanentes que requieren los alumnos en algún momento de su vida escolar a consecuencia de un trastorno o discapacidad diagnosticada por un profesional competente y que necesitan de ayudas y apoyos extraordinarios para acceder o progresar en el currículum por un determinado período de su escolarización. (art. 2, p. 4).

Asimismo, señala que el trastorno de déficit atencional con y sin hiperactividad (TDA) o trastorno hipercinético, coeficiente intelectual(CI) en el rango límite, con limitaciones significativas en la conducta adaptativa, trastornos específicos del lenguaje (TEL), y dificultades específicas del aprendizaje se agrupan bajo el concepto de NEE de carácter no permanente.

\section{Metodología}

Este estudio se adscribe a la metodología cuantitativa, se focaliza en la descripción de las respuestas educativas en la atención a la diversidad por parte de los establecimientos escolares públicos (variable contexto escolar) que participan del Programa de Integración Escolar, desde la perspectiva de los profesionales especialistas de apoyo que se desempeñan en esos establecimientos, en el marco de la legislación educativa vigente. Por tanto, es una investigación no experimental, ex post facto (Briones, 2002) y de carácter micro sociológico. Por la amplitud del trabajo de campo, la recolección de datos se realiza con fuentes mixtas y su profundidad alcanza un grado descriptivo y explicativo.

La recolección de datos se realizó a través de un cuestionario con escala de valoración de 1 al 6 en los conceptos de Nada o Nunca, Poco, Algunas veces, Frecuentemente, Bastante y Siempre, además de respuesta dicotómica frente a algunos indicadores de existencia. Las dimensiones contempladas en el instrumento son las de recursos, escuela o liceo, intervención en la escuela o liceo, currículo, diversidad y diagnóstico, siguiendo a Biencinto et al. (2009). Este cuestionario se sometió a validación 
de contenido por parte de profesionales expertos, y se obtuvo un índice de confiabilidad de 0,89. Luego de su aplicación se obtuvo un nivel de fiabilidad del 0,92, calculado con alfa de Cronbach.

Los agentes participantes son los profesionales especialistas que brindan apoyo a niños, niñas y jóvenes que presentan necesidades educativas especiales de carácter transitorio y permanente, así como los docentes de aula y familias. Trabajan en 30 establecimientos educacionales del nivel básico y medio de las comunas de Curicó y Talca, de los sectores urbano y rural, que cuentan con programa de integración escolar.

De este modo la muestra la conforman en total 92 profesionales especialistas. De ellos el 81,5\% (75) se desempeñan en colegios del área urbana y el 18,5\% (17) lo hace en el área rural de ambas comunas.

El 10,9\% de los profesionales son de género masculino y el $89,1 \%$ de género femenino. Su edad promedio es 36 años, con rangos entre 23 y 60 años. Presentan una media de 10 años de experiencia laboral con una desviación estándar de 10,5 y con un rango mínimo de 1 y máximo de 38 años. Llevan en promedio 4 años trabajando en la Unidad Educativa y con rangos extremos de 1 y 35 años.

Con respecto al punto de vista de la formación profesional (véase la tabla 1), se puede señalar que el $48,9 \%$ (45) de ellos son profesores de educación especial, y conforman el $63,1 \%$ junto a los psicopedagogos; y el 36,9\% queda integrado por psicólogos, fonoaudiólogos, kinesiólogos, trabajadora social y orientadora familiar.

Tabla 1. Profesionales especialistas en apoyo a la atención a la diversidad

\begin{tabular}{lcc}
\hline Profesionales especialistas & F & $\%$ \\
\hline Profesor de Educación Especial & 45 & $48,9 \%$ \\
Psicopedagogo & 13 & $14,2 \%$ \\
Psicólogo & 12 & $13,0 \%$ \\
Fonoaudiólogo & 9 & $9,8 \%$ \\
Kinesiólogo & 5 & $5,4 \%$ \\
Orientadora familiar & 2 & $2,2 \%$ \\
Trabajadora social & 6 & $6,5 \%$ \\
Total & 92 & $100,0 \%$ \\
\hline
\end{tabular}

Fuente: elaboración propia. 
Es importante que los profesionales de apoyo cuenten con formaciones que complementen su especialización general profesional. Los resultados indican que el $70,7 \%$ de ellos cuenta con el grado de licenciatura y el $58,7 \%$ ha realizado estudios posgraduales; el 85,9\% (79) cuenta con una preparación específica en el área de la atención a la diversidad.

En relación con temas particulares de las NEE permanentes se pueden ver los resultados en la tabla 2 , donde se distingue una mayor preparación en el área de la discapacidad intelectual con un 54,3\%.

En toda sociedad las personas en situación de discapacidad presentan dificultades en el ámbito académico, como consecuencia de las barreras y obstáculos que afectan su acceso, permanencia y egreso del sistema escolar.

La Clasificación Internacional del Funcionamiento, de la Discapacidad y de la Salud (CIF) define la discapacidad como

Un término genérico que abarca deficiencias, limitaciones de la actividad y restricciones a la participación. Se entiende por discapacidad la interacción entre las personas que padecen alguna enfermedad (por ejemplo, parálisis cerebral, síndrome de Down y depresión) y factores personales y ambientales (por ejemplo, actitudes negativas, transporte y edificios públicos inaccesibles y un apoyo social limitado). (OMs, 2014).

Tabla 2. Formación específica recibida en NEE permanentes

\begin{tabular}{lcc}
\hline NEE & $F$ & $\%$ \\
\hline Discapacidad motora & 29 & $31,5 \%$ \\
Discapacidad visual & 12 & $13,0 \%$ \\
Discapacidad auditiva & 22 & $23,9 \%$ \\
Discapacidad relación y comunicación & 35 & $38,0 \%$ \\
Discapacidad intelectual & 50 & $54,3 \%$ \\
\hline
\end{tabular}

Fuente: elaboración propia.

Desde una perspectiva médica, el concepto de discapacidad se comprende como

Un término general que abarca las deficiencias, las limitaciones de la actividad y las restricciones de la participación. Las deficiencias son problemas que afectan a una estructura o función corporal; las limitaciones de la actividad son dificultades para ejecutar acciones o tareas, y las restricciones de la participación son problemas para participar en situaciones vitales. (OMS, 2015). 
Así, se observa que la situación de discapacidad de una persona es el resultado de procesos de interacción que realiza con su entorno, basado en parámetros o indicadores limitados a una realidad y descontextualizados de las circunstancias específicas de un individuo en particular. Es por ello que, en la actualidad, se están realizando adaptaciones y acomodaciones diversas para una plena participación de todas las personas, en todos los ámbitos, asegurando desarrollos y aprendizajes para una mejor calidad de vida.

La tabla 3 muestra la preparación de estas profesionales en temas específicos respecto de las NEE transitorias. De manera global, un 49,8\% ha recibido formación en diversas áreas, lo que favorece el apoyo que brindan a nivel de aula regular.

Tabla 3. Formación específica recibida en NEE transitorias

\begin{tabular}{lcc}
\hline NEE & $F$ & $\%$ \\
\hline Trastornos específicos del lenguaje & 51 & $55,4 \%$ \\
Coeficiente intelectual límite & 49 & $53,3 \%$ \\
Déficit atencional & 52 & $56,5 \%$ \\
Dificultades específicas del aprendizaje en lectura & 50 & $54,3 \%$ \\
Dificultades específicas del aprendizaje en escritura & 49 & $53,3 \%$ \\
Dificultades específicas del aprendizaje en matemática & 48 & $52,2 \%$ \\
\hline
\end{tabular}

Fuente: elaboración propia.

Estos profesionales se desempeñan en diversas modalidades de atención educativa: el 51,1\% realiza apoyos especializados en el aula de recursos, el 4,3\% solo apoya en el aula común, el 23,9\% realiza apoyo en aula común y aula de recursos y el 20,7\% comparte apoyo específico en aula de recursos y en aula hospitalaria, donde también efectúan apoyos a domicilio.

Este tipo de atención obedece claramente al concepto de integración, que en palabras de Booth (1996) se comprende como un proceso consistente en responder a la diversidad de necesidades de todos los estudiantes y satisfacerlas mediante una mayor participación en el aprendizaje, las culturas y las comunidades, así como en reducir la exclusión dentro de la educación y a partir de ella.

De este mismo modo, Borsani y Gallicchio (2000) señalan que

Se puede integrar a partir del momento en que se estima que el niño se encuentra en condiciones de ser sujeto activo del proceso de aprendizaje sistemático y de alcanzarlos objetivos escolares propuestos, intentarlo prematuramente o a destiempo puede condenarlo al fracaso. (p. 33). 


\section{Resultados}

A continuación se presentan los resultados a partir del análisis de cada una de las dimensiones por las que se indagó a través del cuestionario aplicado.

\section{Dimensión Recursos}

Los profesionales especialistas declaran que las plazas son ocupadas de manera preferente (89\%) por personas capacitadas para intervenir con los estudiantes con discapacidad, es decir, cuentan con la titulación correspondiente. Además, desde su formación se encuentran preparados para responder y apoyar adecuadamente a los docentes de aula que atienden a una diversidad de estudiantes que se encuentran en los diferentes cursos de los establecimientos educacionales.

Ellos realizan apoyo en el aula común con una alta frecuencia (85,8\%) en asignaturas como Lenguaje y Comunicación, y Matemáticas. Asimismo, cuentan en sus colegios con aulas de recursos o de apoyo pedagógico para brindar acciones educativas específicas relacionadas con el lenguaje oral y escrito, aspectos de matemática y de resolución de problemas. Sin embargo, en un porcentaje menor $(18,5 \%)$ se aplican estrategias para mejorar las competencias lingüísticas de todos los estudiantes.

Lo anterior permite visualizar que las unidades educativas no consideran en la organización técnico-pedagógica a los profesionales de apoyo como un recurso para mejorar los aprendizajes escolares. En consecuencia, no brindan los espacios educativos y los tiempos requeridos para la aplicación de estrategias específicas en los niveles educativos correspondientes.

Los participantes declaran (64,1\%) que los profesores de aula regular agrupan a los alumnos en función de los distintos niveles de rendimiento. Esto evidencia prácticas pedagógicas diferenciadoras que no permiten a los estudiantes "aprender de y con el otro". Además, observan una baja frecuencia (48,9\%) en la realización de acciones tutoriales para acoger e integrar a todos aquellos estudiantes que presentan alguna NEE.

De este modo, se refleja nuevamente la baja o escasa realización de acciones conducentes a evitar el fracaso escolar, tales como los programas de apoyo, programas para el ocio y el tiempo libre. Esto coincide con la falta de sistematicidad en la elaboración e implementación de proyectos de innovación educativa de carácter permanente en el tiempo.

En este sentido, se observa un menor compromiso de parte de los directivos de los establecimientos escolares, al no aprovechar el recurso profesional especializado en educación como en áreas afines -el promedio por escuela es 3,6- para plantear e implementar proyectos innovadores y utilizar de manera eficiente y eficaz los recursos que ofrece el Estado chileno. 
Un factor relevante en esta dimensión es la presencia del recurso humano especializado en cada uno de los centros educativos, los que de manera mayoritaria $(92,4 \%)$ declaran apoyar a los profesores de aula en la atención a los estudiantes con NEE, con una frecuencia del 69,6\%. Asimismo, solo 8 profesionales declaran realizar el servicio de intérpretes (lenguaje de señas) en aulas comunes, de establecimientos de educación media.

En esta misma línea, se observa un porcentaje menor de especialistas $(10,9 \%)$ que realizan apoyo educativo domiciliario a educandos cuyo estado de salud requiere largos periodos de convalecencia.

En la mitad de los colegios participantes del estudio, señalan la existencia de acciones educativas apoyadas por estudiantes ayudantes del mismo curso o de un curso superior, que van en directo beneficio de los educandos que presentan NEE. Es preciso contar con la disposición del profesor del aula regular para implementar estos apoyos con mayor frecuencia.

Los establecimientos escolares presentan bajos niveles de vinculación $(32,6 \%)$ con el entorno y con centros externos tales como organizaciones no gubernamentales y fundaciones, lo que afecta la obtención de recursos y servicios gratuitos para toda la comunidad educativa. De este modo, se observa a la escuela como una institución carente de sentido de pertenencia e identidad con su entorno inmediato, y muy cerrada a la comunidad en general.

Se debe destacar que, de acuerdo con la organización escolar, los directores de los establecimientos educacionales deben realizar acciones de vinculación con la comunidad.

\section{Dimensión Escuela/liceo}

Un porcentaje mayoritario de profesionales especialistas $(69,6 \%)$ señala que los colegios y liceos donde se desempeñan siguen la filosofía de la co-educación y que existen atenciones educativas diferenciadas (93,5\%), en virtud de las cuales adaptan a cada estudiante métodos y estrategias educativas. Asimismo, la respuesta educativa que entregan a los estudiantes está dada en función de sus capacidades $(83,5 \%)$, resaltando que sus integrantes trabajan para proporcionar oportunidades de aprendizaje y para que todos los estudiantes logren sus objetivos. También señalan que se orienta a los alumnos a opciones profesionales o académicas diferentes de acuerdo con sus capacidades (92,4\%).

Otro aspecto importante de mencionar es la participación de la comunidad educativa de un proyecto común y una organización inclusiva para que todos los estudiantes logren los objetivos (81,5\%). Es decir, las escuelas y liceos cuentan con un Proyecto Educativo Institucional enmarcado en las normativas educacionales vigentes y en la realidad propia de la escuela. 


\section{Intervención de la escuela o liceo frente a la diversidad}

Respecto de la respuesta educativa en la atención a la diversidad, la percepción de los profesionales participantes del estudio califica con un 97,8\% la importancia de integrar a los educandos que presentan NEE con la finalidad de adaptarlos al sistema educativo. Además, declaran (72,9\%) que los estudiantes con NEE se agrupan en función de las diferencias en el nivel de aprendizaje escolar en las áreas de lectura, escritura y matemática, y se entregan apoyos y adaptaciones pertinentes (88\%). Del mismo modo, afirman que los estudiantes se agrupan de manera flexible en función del objetivo de la actividad, alternando los grupos para mejorar sus aprendizajes (85,9\%).

\section{Dimensión Currículo}

En relación con esta dimensión, el 83,6\% de los participantes expresa que se aplica el mismo currículo para todos los estudiantes, realizando las adaptaciones necesarias para obtener unos objetivos mínimos. Además indican que a partir del currículo central se crean diferentes itinerarios $(71,7 \%)$.

Lo anterior coincide con lo planteado por el Mineduc al señalar que a partir del año 2017 las escuelas regulares y de educación especial deben trabajar solo con el currículo escolar nacional del nivel de pre-básica y de educación básica, de manera gradual. Se elimina de este modo la existencia de currículos paralelos (Decreto Supremo n. ${ }^{\circ} 83$ del 2015).

También se observan modalidades de atención diferenciadas: una de ellas es el otorgamiento de apoyo específico en el aula de recursos $(60,9 \%)$, otra que los profesionales destacan es la entrega de apoyo especializado $(92,4 \%)$ a los estudiantes diferentes en el aula común junto a su grupo curso. En este sentido, se observa un aumento significativo de la realización de soportes específicos profesionales en las escuelas y liceos.

El $82,6 \%$ de los participantes declara que los currículos se adaptan a las necesidades puntuales de las situaciones gracias al trabajo cooperativo que efectúan los equipos multidisciplinares. Asimismo, el 93,5\% percibe que la comunidad educativa asume la atención de los estudiantes con discapacidad, que se contempla en el proyecto educativo y curricular del colegio o liceo.

\section{Dimensión Diagnóstico}

Ellos destacan en un 96,7\% la importancia del diagnóstico para identificar las diferencias y poder así ajustar la respuesta educativa. El 84,8\% señala que es fundamental diagnosticar para distinguir entre estudiantes dentro de la norma y aquellos que requieren de apoyos específicos, así como la importancia de agruparlos para brindar atención en directa relación con sus necesidades educativas $(93,5 \%)$. 
Destacan en un 99,9\% la necesidad de identificar las aptitudes y los estilos que permitirán a los estudiantes lograr los objetivos de aprendizaje, seleccionando las respuestas educativas más pertinentes a través de la utilización de estrategias docentes adecuadas a sus realidades concretas. Por tanto, señalan en un 79,4\% que realizan diagnósticos basados en sus capacidades cognitivas básicas; sin embargo, desde una perspectiva más clínica, 89,2\% señala que los diagnósticos deben efectuarse desde las necesidades cognitivas y actitudinales que muestran los educandos.

De este modo, los profesionales especialistas transitan entre paradigmas clínicos y curriculares, para enfrentar los procesos de diagnósticos. Lo anterior puede comprenderse por la diversidad en sus formaciones de base, que oscilan de manera preferente entre los ámbitos de la salud y la educación

\section{Dimensión Diversidad}

Con relación al último concepto indagado, el 96,7\% de los profesionales especialistas que se desempeñan en la educación regular señalan que la diversidad es algo positivo y enriquecedor, e indican en un 98,9\% que las diferencias son individuales. Además, el 95,7\% explica que los tipos de diversidad se crean en función de las necesidades educativas que presentan los estudiantes, y que la unidad educativa asume en un 94,5\% como propia las necesidades de esta población.

De este modo, dejan de manifiesto que los profesionales de apoyo visualizan el concepto de diversidad de una manera restringida y solo relacionado con las NEE.

\section{Conclusiones}

Con respecto a la respuesta educativa de los 92 profesionales de apoyo al sistema escolar en relación con la variable contexto escolar y en el marco del Programa de Integración Escolar, se puede señalar que reconocen la existencia de la diversidad como algo propio de los seres humanos, ya que las diferencias son individuales y se crean en función los requerimientos particulares. Otorgan los mismos derechos y deberes a todo estudiante, independientemente de sus características personales, a través de la variedad de experiencias de aprendizaje que brindan cuando entregan apoyo en el aula regular. Además, evidencian que las necesidades de cada estudiante son para la escuela o liceo un desafío y una responsabilidad; es decir,

Bajo el paradigma de la inclusión la comunidad educativa acepta a todos los discentes sin plantearse ningún tipo de segregación y las necesidades de cada uno de ellos se convierten en prioridades del 
centro educativo en su conjunto, optando por el desarrollo de proyectos encaminados hacia ideales comunes en materia de equidad. (Arnaiz y Azorín, 2014, p. 4).

Los profesionales de apoyo consideran los establecimientos escolares donde se desempeñan como instituciones que están movilizándose hacia prácticas educativas cada vez más inclusivas, a través del apoyo de especialistas en áreas específicas y la permanente colaboración de la familia. Sin embargo, se requiere de mayor tiempo para modificar, integrar y asumir conductas más abiertas a la diversidad.

Además, evidencian que las necesidades que presentan los estudiantes son para la escuela o liceo un desafío y una responsabilidad, porque deben cautelar y asegurar los aprendizajes y la participación de cada uno de ellos, independientemente de su condición. Para ello deben organizar la respuesta educativa como toma de decisión curricular, a partir de procesos de evaluación que deben incluir áreas o aspectos más transversales, como por ejemplo las actitudes, motivaciones e intereses, considerando apoyos y servicios específicos en diversos contextos de participación y cooperación mutua.

Como aspectos por mejorar, se destaca el brindar más apoyo a los docentes de aula en temas atingentes a sus especialidades, para que puedan efectivamente ajustar la respuesta educativa en el aula, de tal modo que todos los estudiantes se vean beneficiados por la aplicación de metodologías, estrategias, recursos variados y contextualizados a la realidad específica de cada curso.

Lo anterior indica el replantear el trabajo colaborativo que se está desarrollando en los establecimientos educacionales, entre los docentes de aula y los profesionales de apoyo. Se deben realizar sesiones de intercambio de conocimientos de aspectos técnicos y pedagógicos, y de cómo se operacionalizan en beneficio directo de los niños, niñas y jóvenes con y sin NEE para fomentar y fortalecer procesos de inclusión.

Se observa también, a partir de sus explicitaciones, que ha bajado el nivel de apoyo que se entrega en el aula de recursos y ha aumentado de manera significativa el ingreso por parte de ellos al aula común, lo cual favorece procesos de co-educación en el marco del trabajo colaborativo. Se destaca que estos cambios han ido sucediendo de manera paulatina, como respuesta a las demandas de las políticas y normativas educacionales propias del contexto nacional.

Estas prácticas pedagógicas integradas requieren de tareas colaborativas, relacionadas con la toma de decisiones, la distribución de tareas, la selección e implementación de estrategias de enseñanza y aprendizaje diversificadas. Todo lo anterior favorece las probabilidades de éxito y del logro de objetivos de aprendizaje de todos los educandos y de manera más específica de aquellos que presentan NEE. 
Una posible línea de investigación futura es indagar respecto de la mejora en los procesos de aprendizaje de los educandos, que debiesen traducirse en un impacto positivo en la calidad y equidad educativa.

Finalmente, se concluye que los procesos pedagógicos deben ir adaptándose a las nuevas demandas que se presentan en las aulas regulares y en concordancia con la formación inicial de los profesores, enmarcándose en las declaraciones, políticas y legislaciones propias del país, así como de las diversas organizaciones internacionales que señalan "la educación como un derecho". Se responde de esta manera a la diversidad educativa existente en nuestras escuelas y liceos.

\section{Referencias}

Asensio, I.; Biencinto, C., García Corona, D., García Nieto, N., Mafokozi, J. y Ramos, J. (2005/2006). Innovación y eficacia en atención a la diversidad en Educación Secundaria Obligatoria: ¿Qué hace que las experiencias de atención a la diversidad funcionen en los centros de la Comunidad Autónoma de Madrid? Contextos Educativos, 8-9, 15-32.

Arnaiz, P. y Azorín, C. (2014). Autoevaluación docente para la mejora de los procesos educativos en escuelas que caminan hacia la inclusión. Revista Colombiana de Educación [internet], 67, 227-247. Recuperado de http:www.scielo.org.co/pdf/rcde/n67/n67a11.pdf

Beeny, K. (1975). Una experiencia de integración educativa. Siglo Cero, 42, 33-38.

Biencinto-López, Ch.; González-Barbera, C.; García-García, M.; Sánchez-Delgado, P. y Madrid-Vivar, D. (2009). Diseño y propiedades psicométricas del Avaco-Evadie. Cuestionario para la evaluación de la atención a la diversidad como dimensión educativa en las instituciones escolares. Relieve, 15 (1), 1-36. Recuperado de http://www. uv.es/RELIEVE/v15n1/RELIEVEV15n1_4.htm.

Booth, T. (1996). A perspective on inclusion from England. Cambridge Journal of Education, 2(1), 87-99.

Booth, T. (2002). Inclusión y exclusión en la ciudad: conceptos y contextos. Londres, Inglaterra: Routledge.

Booth, T. y Ainscow, M. (2000). Índice de inclusión: desarrollando el aprendizaje y la participación en las escuelas. ISBN: 84-607-5734-x.

Borsani, M. y Gallicchio, M. (2000). Integración o exclusión: la escuela común y los niños con necesidades educativas especiales. Buenos Aires, Argentina: Novedades Educativas.

Briones, G. (2002). Metodología de la investigación cuantitativa de las ciencias sociales. Instituto Colombiano para el Fomento de la Educación 
Superior. Recuperado de http://200.14.205.40:8080/portalicfes/ home_2/rec/arc_77.pdf

Cabrerizo, J. y Rubio, M. (2007). Atención a la diversidad. Madrid, España: Prentice- Hall.

Cardona, M. (2006). Diversidad y educación inclusiva: enfoques metodológicos estrategia para una enseñanza colaborativa. Madrid, España: Pearson.

Fragoso, E. y Canales, E. (2009). Estrategias educativas para la formación en valores desde la educación informal de la familia. Educere [internet], 13(44) 177-185. Recuperado de http://www.scielo.org.ve/pdf/ edu/v13n44/art21.pdf.

Jiménez, C. (2005). Pedagogía diferencial: diversidad equidad. Madrid, España: Pearson.

López, M. (2004). Construyendo una escuela sin exclusiones: una forma de trabajar en el aula con proyectos de investigación. Málaga, España: Aljibe.

Ministerio de Educación de Chile, Mineduc (2009). Decreto n. ${ }^{\circ} 170$ Fija normas para determinar los alumnos con necesidades educativas especiales que serán beneficiarios de las subvenciones para educación especial.

Ministerio de Educación de Chile, Mineduc (2009). Ley de Educación General n. ${ }^{\circ} 20.370$.

Ministerio de Educación de Chile, Mineduc (2014). Manual de orientaciones y apoyo a la gestión (directores y sostenedores). Programa de Integración Escolar (PIE). Decreto Supremo 170 del 2009.

Ministerio de Educación de Chile, Mineduc (2015). Decreto Exento n. ${ }^{\circ}$ 83 Aprueba criterios y orientaciones de adecuación curricular para estudiantes con necesidades educativas especiales de educación parvularia y educación básica.

Ministerio de Educación del Perú (2006). Manual de educación inclusiva. Recuperado de http://www.oei.es/pdfs/educacion_inclusiva_peru.pdf.

Organización de las Naciones Unidas para la Educación, la Ciencia y la Cultura, Unesco (2001). Declaración universal sobre la diversidad cultural. París: Francia. Recuperado de http://unesdoc.unesco.org/ images/0012/001271/127162s.pdf.

Organización Mundial de la Salud, oms (2014). Discapacidad y salud. Nota descriptiva n. ${ }^{\circ} 352$. Recuperado de http://www.who.int/mediacentre/facsheets/fs352/es/

Organización Mundial de la Salud, OMS (2015). Conceptualización de discapacidad. Recuperado de http://www.who.int/topics/disabilities/es/

Ruiz, P. (2010). La evolución de la atención a la diversidad del alumnado de educación primaria a lo largo de la historia. Temas para la Educación: Revista digital para profesionales de la enseñanza, 8, 1-15.

Salinas, G. (2007). Atención a la diversidad. Necesidades educativas: guía de actuación para docentes. Santiago, Chile: Ideas propias. 